\title{
Morning Mastication Enhances Postprandial Glucose Metabolism in Healthy Young Subjects
}

\author{
Arisa Sato, ${ }^{1,2}$ Yoshinori Ohtsuka ${ }^{3}$ and Yujiro Yamanaka ${ }^{4,5}$ \\ ${ }^{1}$ School of Education, Hokkaido University, Sapporo, Hokkaido, Japan \\ ${ }^{2}$ School of Medicine, Asahikawa Medical University, Asahikawa, Hokkaido, Japan \\ ${ }^{3}$ Department of Sports and Human Studies, Sapporo International University, Sapporo, Hokkaido, Japan \\ ${ }^{4}$ Research and Education Center for Brain Science, Hokkaido University, Sapporo, Hokkaido, Japan \\ ${ }^{5}$ Laboratory of Life and Health Sciences, Graduate School of Education, Hokkaido University, Sapporo, \\ Hokkaido, Japan
}

\begin{abstract}
Postprandial glucose concentration is dependent on the time of day and its concentration in the morning is lower than in the evening. However, whether it is dependent on mastication at different times of the day has not been studied before. We hypothesized that mastication affects insulin-mediated glucose metabolism differently in the morning and evening in healthy individuals. Firstly, nine healthy male volunteers $\left(22.0 \pm 0.7\right.$ SEM years, body mass index $\left.22.0 \pm 1.0 \mathrm{~kg} / \mathrm{m}^{2}\right)$ performed a $75-\mathrm{g}$ oral glucose tolerance test (OGTT). One week after the OGTT, they participated in a high-carbohydrate food (rice) consumption test with 10 or 40 chews per mouthful. Each experiment was conducted in the morning (0800 $\mathrm{h})$ and evening $(2000 \mathrm{~h})$ on the same day. Blood samples were collected before and at 30 -min intervals for 120 min after glucose or rice consumption. The incremental area under the curve (iAUC) for glucose in the OGTT was significantly lower in the morning than in the evening, whereas the iAUC for insulin was similar at both times. In participants who chewed 40 times, the iAUC for glucose after rice consumption was significantly lower in the morning than in the evening but was similar at both times in individuals who chewed 10 times. Chewing 40 times in the morning (but not the evening) significantly increased insulin secretion at $30 \mathrm{~min}$. This suggests that morning mastication improves early-phase insulin secretion after rice consumption. This novel finding may aid in reducing the incidence of obesity and type 2 diabetes mellitus.
\end{abstract}

Keywords: circadian rhythm; early-phase insulin secretion; morning mastication; postprandial glucose metabolism; type2 diabetes

Tohoku J. Exp. Med., 2019 November, 249 (3), 193-201.

(C) 2019 Tohoku University Medical Press

\section{Introduction}

Epidemiological studies associate mastication and meal duration with diabetes and obesity and body composition (Takayama et al. 2002; Maruyama et al. 2008; Ohkuma et al. 2013; Yamane et al. 2014). It is generally accepted that thorough chewing of food and slow eating have the potential to decrease the risk of diabetes and obesity.

Increased number of chew and/or thorough chewing has been reported to alter the secretion of hormones related to appetite and energy metabolism. Cassady et al. (2009) demonstrated that healthy participants who chewed test food (55 g of almonds) 40 times had lower postprandial glucagon-like peptide 1 (GLP1) levels than those who chewed 10 or 25 times. Li et al. (2011) reported that both lean and obese participants who chewed 40 times had lower energy intake and postprandial ghrelin levels and higher postprandial GLP1 and cholecystokinin levels than those who chewed 15 times. Zhu et al. (2014) found that mastication improved insulin-mediated glucose metabolism in older men; older participants who chewed test food 40 times had higher levels of plasma glucose, insulin, and glucose-dependent insulinotropic peptide at meal completion than those who chewed 15 times. According to other studies, mastication also influences postprandial satiety, appetite, and diet-induced thermogenesis (Hetherington and Boyland 2007; Hetherington and Regan 2011; Mattes and Considine 2013; Zhu and Hollis 2014; Hamada et al. 2014, 2016; Komai et al. 2016).

Regarding the circadian rhythm of glucose metabolism, glucose tolerance and insulin sensitivity are higher in the morning than in the evening, as shown in a study with healthy and prediabetic participants aged 15-19 years (Jarrett and Keen 1969). Glucose concentrations are higher

Received September 24, 2019; revised and accepted November 6, 2019. Published online November 23, 2019; doi: 10.1620/tjem.249.193.

Correspondence: Yujiro Yamanaka, Laboratory of Life and Health Sciences, Graduate School of Education, Hokkaido University, North-11 West-7, Kita-Ku, Sapporo, Hokkaido 060-0811, Japan.

e-mail: y-yu2ro@edu.hokudai.ac.jp 
in oral glucose tolerance tests (OGTTs) conducted in the afternoon and evening than in those conducted in the morning (Jarrett et al. 1972; Carroll and Nestel 1973), whereas insulin secretions are higher and increase more rapidly in the morning (Aparicio et al. 1974; Zimmet et al. 1974; Sonnier et al. 2014). Insulin secretions tend to be lowest in the afternoon and evening, with a delayed rise and late peak response, as is typically seen in diabetes (Aparicio et al. 1974; Zimmet et al. 1974; Sonnier et al. 2014). In addition, diurnal variations in glucose and insulin responses to a mixed meal are consistent with those in OGTTs (Ahmed et al. 1976; Nuttall et al. 1985).

To our knowledge, no previous studies have evaluated the effect of time-of-day-specific mastication on postprandial glucose metabolism in healthy participants. There are two possible hypotheses that mastication could increase insulin-mediated glucose metabolism. The first is that mastication enhances so-called preabsorptive insulin response within a few minutes of ingestion (Teff 2000), which the intraoral sensory stimulation from food elicits insulin secretion through the release of acetylcholine from the vagus nerve to the pancreas (Suzuki et al. 2005). The other is that mastication may increase insulin secretion after eating, since it has been reported that the amount of dietary induced thermogenesis is positively correlated with insulin secretion after eating (Rothwell and Stock 1981; MarquesLopes et al. 2003). Therefore, the purpose of the present study was to assess if mastication differentially improves insulin-mediated glucose metabolism between morning and evening in healthy participants.

\section{Participants and Methods}

Participants

Nine healthy male participants were enrolled in this study as paid volunteers. All participants were recruited through advertising posters and website at Hokkaido University in Japan. All participants were in good physical condition with no personal history of psychiatric, endocrine, or sleep disorders. Their jobs did not require working early in the morning, late at night, or rotational night shifts. The
Morningness-Eveningness questionnaire (MEQ) (Horne and Ostberg 1976) was used to identify individual chronotypes to confirm that participants were not extreme larks or owls. The Pittsburgh Sleep Quality Index (PSQI) (Doi et al. 2000) was used to assess sleep quality.

All participants gave written informed consent before entering the study; this allowed them to withdraw the study whenever they wished. The study protocol was approved by the ethical committee of the Hokkaido University Graduate School of Education (no. 17-40), and the study was conducted in accordance with the Declaration of Helsinki.

\section{Experimental Protocol}

All participants participated in a 75-g OGTT in the morning $(0800 \mathrm{~h})$ and evening $(2000 \mathrm{~h})$ of the same day (Fig. 1). One week after the OGTT, they participated in two high-carbohydrate food (white rice) tests with a crossover design. In these tests, they consumed a fixed amount of carbohydrate-rich food (white rice) in the morning $(0800 \mathrm{~h})$ and evening $(2000 \mathrm{~h})$ on the same day (Fig. 1). In the first test, they chewed the food 10 times; in the second (conducted a week later), they chewed the food 40 times. The order of two experiments was determined by using random allocation software GraphPad Software (GraphPad Software Inc., CA, USA).

One to 2 weeks before the first OGTT, the participants were instructed to maintain a regular sleep schedule, going to sleep between $2300 \mathrm{~h}$ and $0000 \mathrm{~h}$ and waking up between $0700 \mathrm{~h}$ and 0800 h. On the night before each test, the participants were asked to consume a standardized meal (total energy, $261 \mathrm{kcal} ; 85 \%$ carbohydrate; $11 \%$ protein; $4 \%$ fat) before $2100 \mathrm{~h}$ and to fast from $2100 \mathrm{~h}$ until $0800 \mathrm{~h}$.

On the test day, the participants arrived at the laboratory before $0800 \mathrm{~h}$. They rested for 10-20 min and within the next $10 \mathrm{~min}$ were administered either $75 \mathrm{~g}$ of liquid glucose (Ay Pharma Corp, Japan) or white rice (total energy, $370 \mathrm{kcal} ; 92 \%$ carbohydrate, $6 \%$ protein, $2 \%$ fat). Approximately $3 \mathrm{~mL}$ of blood was drawn before consumption $(0 \mathrm{~min})$ and $30,60,90$, and $120 \mathrm{~min}$ thereafter for measurement of blood glucose concentrations and plasma levels. Following the 120-min blood sampling $(1030 \mathrm{~h})$, the participants ate a standardized meal (total energy, $579 \mathrm{kcal} ; 90 \%$ carbohydrate, $8 \%$ protein, $2 \%$ fat). They did not consume any additional food or liquids other than water until the evening test $(2000 \mathrm{~h})$, nor did perform any physical exercise

\section{Time of day $(h)$}

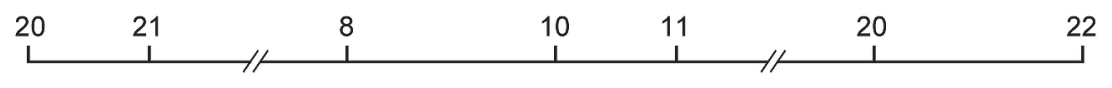

\begin{tabular}{|c|c|c|c|c|c|c|}
\hline Exp 1. OGTT & Meal & Fasting & Morning, OGTT & Meal & Fasting & Evening, OGTT \\
\hline & & & & \multicolumn{3}{|c|}{ One week interval } \\
\hline \multirow[t]{2}{*}{ Exp 2. Chew 10 times } & Meal & Fasting & Morning, Rice & Meal & Fasting & Evening, Rice \\
\hline & & & & \multicolumn{3}{|c|}{ One week interval } \\
\hline Exp 3. Chew 40 times & Meal & Fasting & Morning, Rice & Meal & Fasting & Evening, Rice \\
\hline
\end{tabular}

Fig. 1. Timelines for the OGTTs and high-carbohydrate food tests.

The latter had a crossover design: the participants chewed the food 10 times in the first test (Exp. 2) and 40 times in the second test (Exp. 3).

Exp., experiment; OGTT, oral glucose tolerance test. 
during this interval. The evening test was performed in the same way as the morning test.

\section{Measurements and Analytical Determinations}

Approximately $3 \mathrm{~mL}$ of venous blood was collected through an indwelling intravenous catheter with a heparin lock placed in a forearm vein. The total blood glucose concentration was determined using a quinoprotein glucose dehydrogenase variant-based blood glucose monitoring system (ACCU-CHECK, Roche Diagnostics, Germany). The remaining blood was immediately centrifuged at $4^{\circ} \mathrm{C}$ $(3,000 \mathrm{rpm}, 15 \mathrm{~min})$, and the plasma was frozen at $-30^{\circ} \mathrm{C}$ for all other assays.

The plasma insulin secretion was determined using an ELISA kit (10-1113-01, Mercodia Insulin ELISA, Sweden) with a sensitivity of $6 \mathrm{pmol} / \mathrm{L}$ and intra- and inter-assay coefficients of variation of $5.9 \%$ and $2.0 \%$, respectively. The total plasma GLP1 level was assayed using an ELISA kit (YK161, Total GLP1-HS ELISA Kit, Yanaihara Institute, Japan) with a sensitivity of $0.7 \mathrm{pmol} / \mathrm{L}$ and intraand inter-assay coefficients of variation of $2.2 \%$ and $2.0 \%$, respectively. All assays were performed according to the manufacturer's instructions. Plasma amylase activity was measured using a CicaLiquid-N AMY kit (Kanto Chemical, Japan); the substrate was 2-chloro-4-nitrophenyl-4-galactopyranosylmaltoside, and the measurement was performed in a commercial laboratory (SRL Sapporo, Japan).

\section{Calculations and Statistical Analysis}

The incremental areas under the curve (iAUCs) were calculated for the blood glucose concentrations and plasma insulin secretions during each 120-min test using the trapezoid method. To assess $\beta$-cell function, the insulinogenic index was calculated as follows: (insulin secretion at $30 \mathrm{~min}$ minus fasting insulin secretion) divided by the difference in blood glucose concentration at $30 \mathrm{~min}$ (Seltzer et al. 1967). All data are expressed as mean \pm standard error of the mean (SEM).

To determine whether the effect of mastication on insulin-mediated glucose metabolism differed by time of day, we compared the data from the morning and evening tests. The differences between single values (iAUCs and insulinogenic indexes) in the morning and evening tests were analyzed using Student's $t$ test. Repeated measures analysis of variance (ANOVA) with a post-hoc Bonferroni test was used for comparison of continuous variables. GraphPad Prism version 7 (GraphPad Software Inc., CA) was used for all statistical analyses. A $p$ value $<0.05$ was considered statistically significant.

\section{Results}

\section{Participant Characteristics}

Nine healthy men participated in the present study. All participants completed all tests. The mean age of the participants was $22.0 \pm 0.7$ years, and the mean body mass index was $22.0 \pm 1.0 \mathrm{~kg} / \mathrm{m}^{2}$. The mean MEQ score across all participants was $54.6 \pm 2.1$, and the mean PSQI score was $2.8 \pm 0.6$. No participants were extreme larks (MEQ score $>70$ ), extreme owls (MEQ score < 30) (Horne and Ostberg 1976), or poor sleepers (PSQI score $>5.5$ ) (Doi et al. 2000).
Glucose Concentrations and Insulin Secretions in the Morning and Evening OGTTS

To assess whether or not glucose tolerance shows significant diurnal difference as reported previously (Jarret and Keen 1969; Jarret et al. 1972; Carroll and Nestel 1973), 75-g OGTTs were performed in the morning and evening. ANOVA revealed a significant time-of-day (morning vs. evening) effect on blood glucose concentration $(p<0.001)$, but not plasma insulin secretion $(p=0.384)$, in $75-\mathrm{g}$ OGTTs. Mean fasting glucose concentrations $(0 \mathrm{~min})$ were higher (although not significantly) in the morning $(87 \pm 2$ $\mathrm{mg} / \mathrm{dL})$ than in the evening $(82 \pm 2 \mathrm{mg} / \mathrm{dL})$ (Fig. 2A). Conversely, non-fasting glucose concentrations were significantly lower in the morning than in the evening at $60 \mathrm{~min}$ (132 \pm 8 vs. $156 \pm 8 \mathrm{mg} / \mathrm{dL}, p<0.001), 90 \mathrm{~min}(153 \pm 7$ vs. $125 \pm 5 \mathrm{mg} / \mathrm{dL}, p<0.001)$, and $120 \mathrm{~min}(130 \pm 6$ vs. $107 \pm$ $3 \mathrm{mg} / \mathrm{dL}, p<0.001)$.

The insulin secretion in the morning was significantly higher than that in the evening at $30 \mathrm{~min}(p<0.01)$ (Fig. $2 \mathrm{~B})$. Although fasting and non-fasting blood glucose concentrations at 60,90 , and 120 min were statistically different between the morning and evening tests (Fig. 2A), no significant differences were observed between insulin secretions in the morning and evening at $60 \mathrm{~min}(70.4 \pm 13.6$ and $64.4 \pm 17.9 \mathrm{mU} / \mathrm{L}), 90 \mathrm{~min}(74.8 \pm 15.1$ and $72.5 \pm 22.3$ $\mathrm{mU} / \mathrm{L})$, or $120 \mathrm{~min}(50.3$ and 6.9 vs. $57.0 \pm 9.8 \mathrm{mU} / \mathrm{L})$.

The iAUC for glucose was significantly smaller in the morning than in the evening $(4,285.0 \pm 558.9$ vs. $6,463.3 \pm$ $611.9 \mathrm{mg} \mathrm{h} / \mathrm{dL} ; p<0.01$ ) (Fig. 2C). The iAUC for insulin was higher in the morning than in the evening $(6,556.7 \pm$ $1,116.0$ and 5,686.7 $\pm 1,589.2 \mathrm{mg} \mathrm{h} / \mathrm{dL}$, respectively) (Fig. 2D), as was the insulinogenic index, a marker of earlyphase $\beta$-cell function $(1.32 \pm 0.21$ and $0.98 \pm 0.32 \mathrm{mU} / \mathrm{mg}$, respectively); however, neither difference was significant (both $p$-values $=0.25)$ (Fig. 2E).

Postprandial Glucose and Insulin Responses to Highcarbohydrate Food Consumed in the Morning and Evening

Next, we evaluated the time-of-day effect of mastication on postprandial glucose and insulin responses to white rice. ANOVA showed significant differences between the time of day $(p<0.05)$ and glucose concentrations at various time points $(p<0.01)$ (Fig. 3A). However, the number of chews (10 vs. 40$)$ had no effect on glucose levels, nor did the combination of time of day and number of chews $(p=$ $0.34)$. Time of day $(p=0.19)$, number of chews $(p=0.08)$, and a combination of both variables $(p=0.34)$ did not have a significant effect on plasma insulin secretions (Fig. 3B).

The postprandial glucose levels were lower in participants who chewed 40 times in the morning vs. the evening at $60 \mathrm{~min}(106.9 \pm 4.2$ vs. $135.1 \pm 7.3 \mathrm{mg} / \mathrm{dL}, p<0.01), 90$ $\min (104.1 \pm 4.5$ vs. $126.8 \pm 6.9 \mathrm{mg} / \mathrm{dL}, p<0.05)$, and 120 $\min (100.0 \pm 3.1$ vs. $118.3 \pm 5.3 \mathrm{mg} / \mathrm{dL}, p=0.132)$ even when the ANOVA test showed no significant differences (Fig. 3A). The iAUC for glucose was significantly lower in participants who chewed 40 times in the morning $(2,548.3$ 

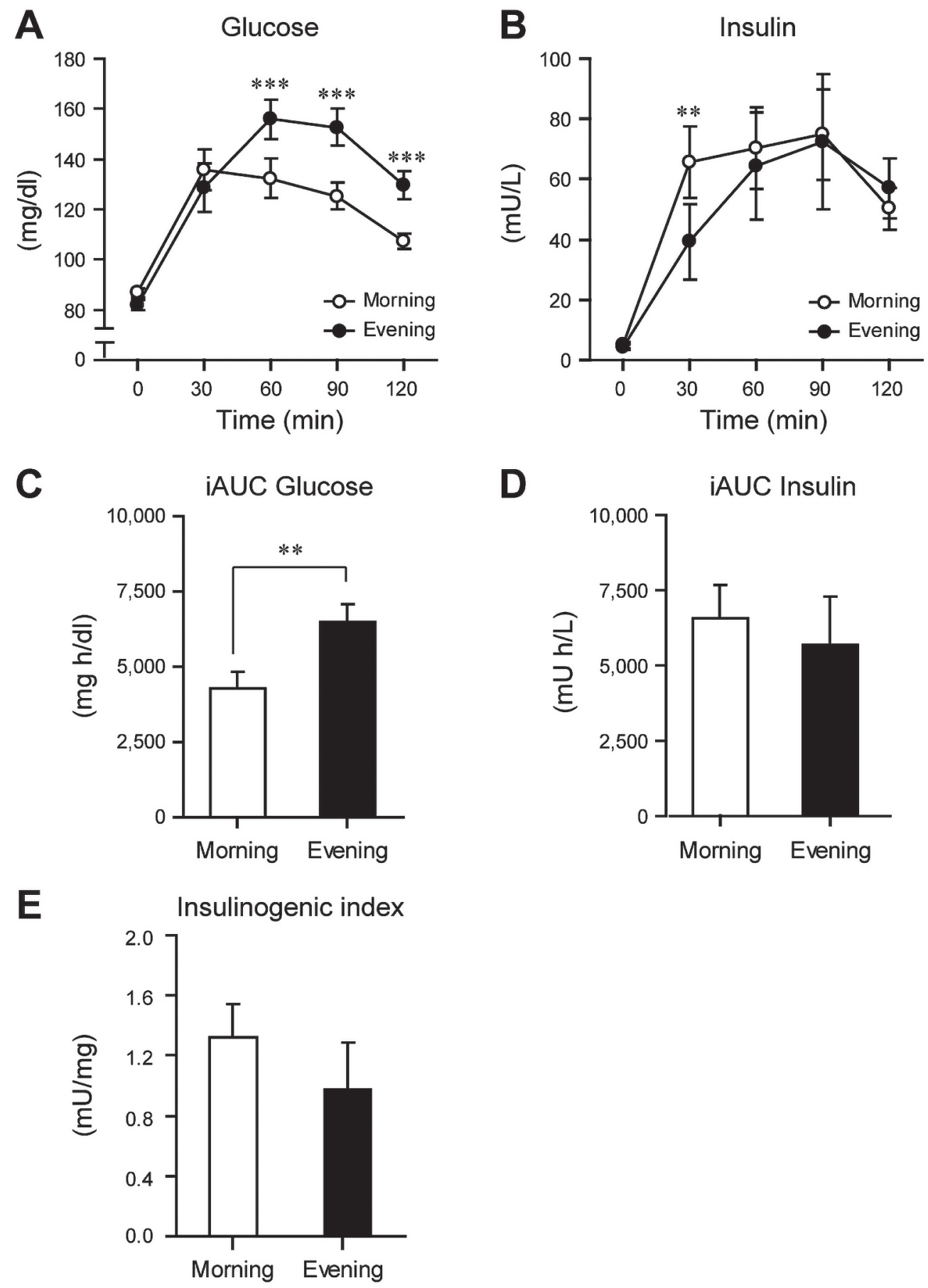

Fig. 2. Comparison of the results of the OGTTs in the morning and evening.

Glucose (A) and insulin (B), iAUCs for glucose (C) and insulin (D), and insulinogenic indices (E) are shown. The insulinogenic index $[\Delta$ insulin $(0-30 \mathrm{~min}) / \Delta$ glucose $(0-30 \mathrm{~min})]$ was calculated from the OGTT results in the morning and evening. Data are represented as mean $\pm \operatorname{SEM}(\mathrm{n}=9)$ and were analyzed using two-way repeated measures ANOVA and post hoc Bonferroni test or the paired $t$ test.

ANOVA, analysis of variance; iAUC, incremental area under the curve; OGTT, oral glucose tolerance test.

${ }^{* *} p<0.01,{ }^{* * *} p<0.001$; morning $(0800 \mathrm{~h})$ vs. evening $(2000 \mathrm{~h})$.

$\pm 342.6 \mathrm{mg} \mathrm{h} / \mathrm{dL}$ ) than in those who chewed either 10 times $(4,428.3 \pm 607.2 \mathrm{mg} \mathrm{h} / \mathrm{dL} ; p<0.05)$ or 40 times $(4,435.0 \pm$ $613.2 \mathrm{mg} \mathrm{h} / \mathrm{dL} ; p<0.01)$ in the evening. It also tended to be lower in participants who chewed 40 times vs. 10 times in the morning $(3,788.3 \pm 522.5 \mathrm{mg} \mathrm{h} / \mathrm{dL})(p=0.06)$ (Fig.
3C).

Insulin secretions at 30 min were significantly higher in participants who chewed 40 times in the morning (50.7 \pm $4.0 \mathrm{mU} / \mathrm{L}$ ) than in those who chewed either 40 times in the evening $(34.4 \pm 4.6 \mathrm{mU} / \mathrm{L}, p<0.05)$ or 10 times in the 
A

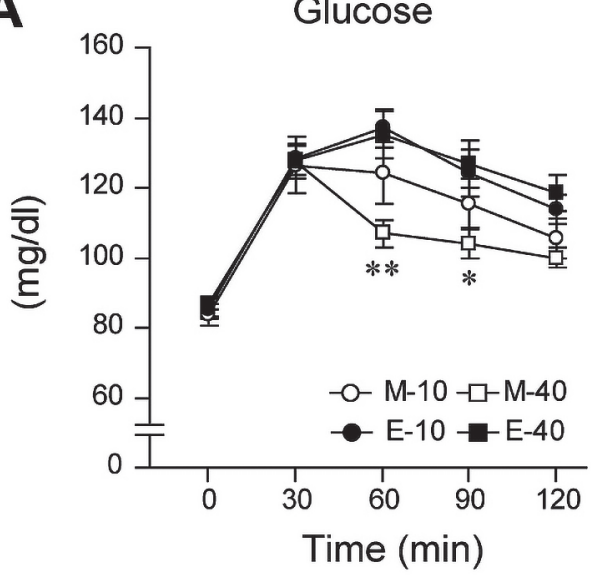

C

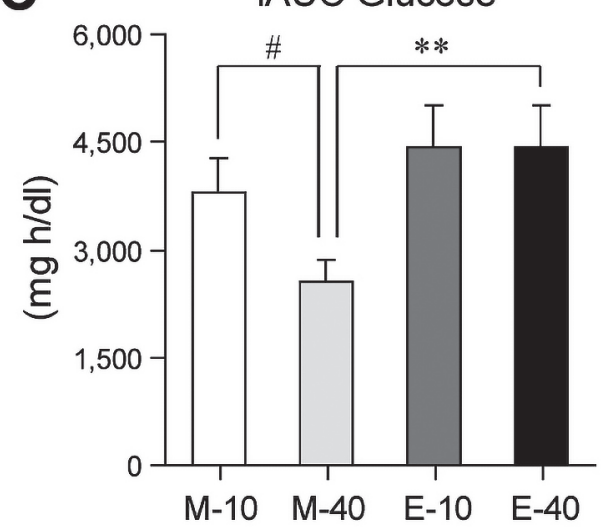

B

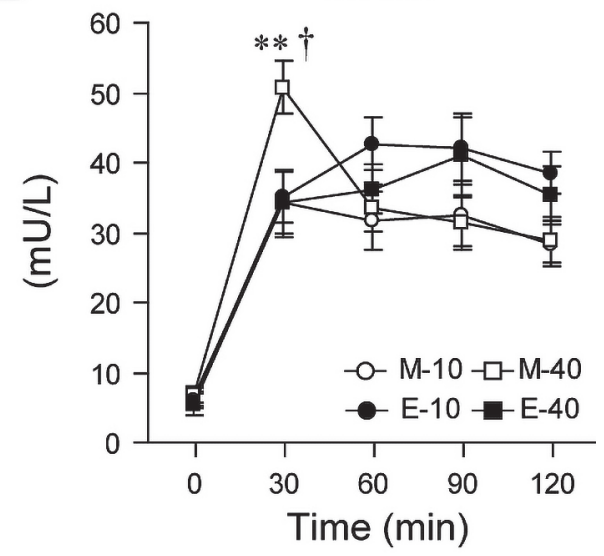

D

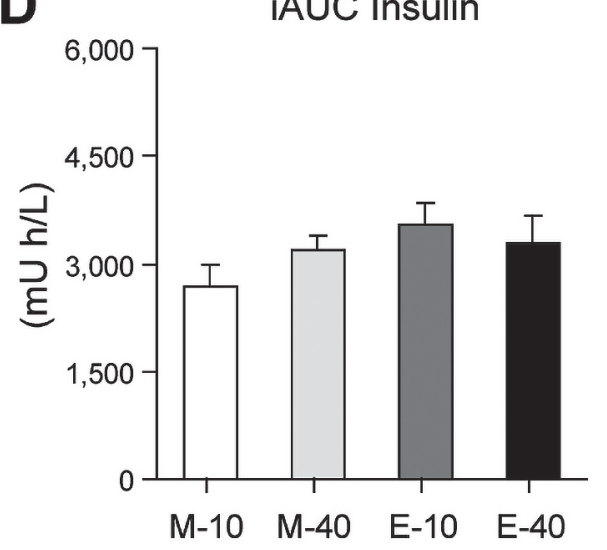

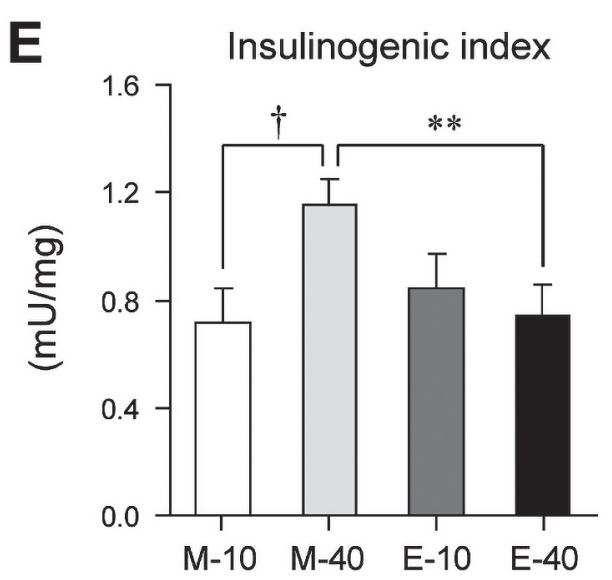

Fig. 3. Effect of mastication on glucose concentrations and insulin secretions following high-carbohydrate food consumption.

Shown are glucose (A), insulin (B), iAUCs for glucose (C) and insulin (D), and insulinogenic indices (E) in the morning and evening. The insulinogenic index [ $\Delta$ insulin $(0-30 \mathrm{~min}) / \Delta$ glucose $(0-30 \mathrm{~min})]$ was calculated from the glucose concentrations and insulin secretions in each condition. Data are represented as mean $\pm \operatorname{SEM}(n=9)$ and were analyzed using two-way repeated measures ANOVA and post hoc Bonferroni test or the paired $t$ test.

ANOVA, analysis of variance; E-10, 10 chews in the evening; E-40, 40 chews in the evening; iAUC, incremental area under the curve; M-10, 10 chews in the morning; M-40, 40 chews in the morning; SEM, standard error of the mean $* p<0.05, * * p<0.01, * * * p<0.001 ; \mathrm{M}-40$ vs. E-40.

$\# p=0.06,{ }^{\dagger} p<0.05 ; \mathrm{M}-10$ vs. M-40. 

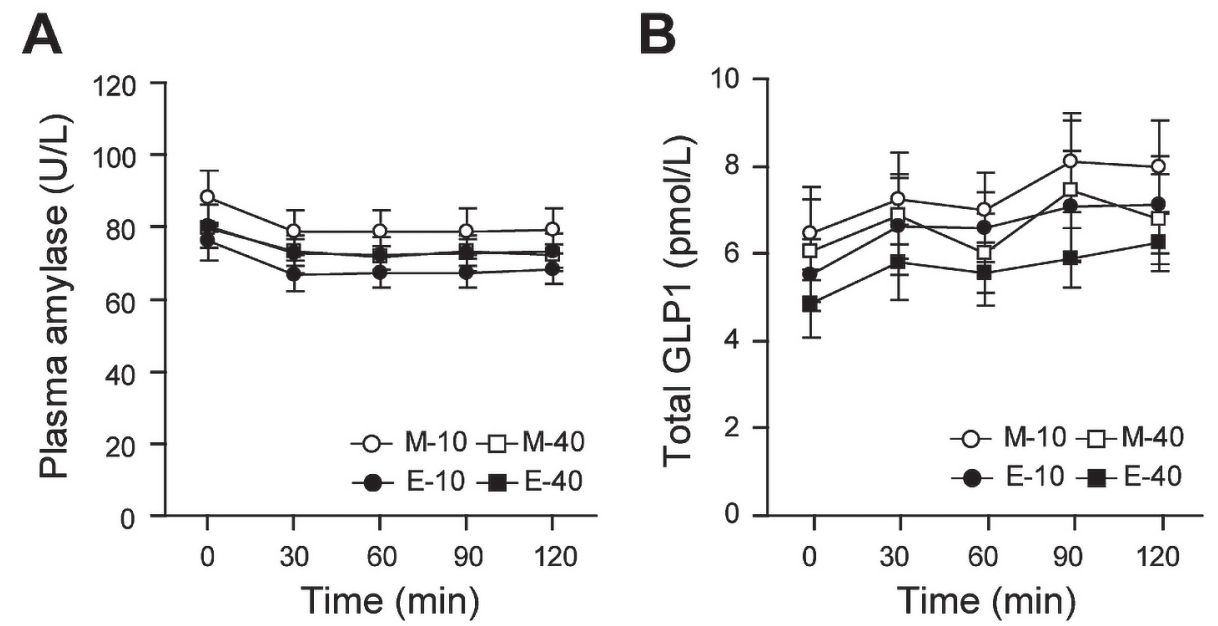

Fig. 4. Effect of mastication on total plasma amylase activity and GLP1 levels following high-carbohydrate food consumption.

Amylase activity (A) and GLP1 levels (B) levels in the morning and evening are shown. Data are represented as mean \pm SEM $(\mathrm{n}=9)$.

E-10, 10 chews in the evening; E-40, 40 chews in the evening; GLP1, glucagon-like peptide 1; M-10, 10 chews in the morning; M-40, 40 chews in the morning; SEM, standard error of the mean.

morning $(34.2 \pm 5.0 \mathrm{mU} / \mathrm{L}, p<0.05)$; nevertheless, the overall ANOVA test was not significant (Fig. 3B). The iAUC for insulin did not differ among the four groups (Fig. $3 \mathrm{D})$. The insulinogenic index was significantly higher in participants who chewed 40 times in the morning $(1.15 \pm$ $0.10 \mathrm{mU} / \mathrm{L})$ than in those who chewed 10 times in the morning $(0.72 \pm 0.13 \mathrm{mU} / \mathrm{L} ; p<0.05)$ and those who chewed 40 times in the evening $(0.74 \pm 0.12 \mathrm{mU} / \mathrm{L} ; p<$ 0.01) (Fig. 3E).

To determine how chewing 40 times in the morning might facilitate $\beta$-cell function, we measured plasma amylase activity (Fig. 4A) and total GLP1 levels (Fig. 4B). Mastication did not significantly affect either of these variables. Hence, the mechanism whereby morning mastication improves early $\beta$-cell function is not involved in amylase activity or GLP1 levels.

\section{Discussion}

Although previous studies (Hetherington and Boyland 2007; Cassady et al. 2009; Li et al. 2011; Hetherington and Regan 2011 ; Mattes and Considine 2013; Zhu and Hollis 2014; Zhu et al. 2014; Hamada et al. 2014, 2016; Komai et al. 2016) have described the effects of mastication on food intake, self-reported appetite (satiety), body composition, postprandial energy expenditure, and secretion of appetiterelated hormones, little is known about the effects of mastication on glucose metabolism and insulin secretion at different times of day. We investigated diurnal variations in glucose metabolism by administering 75-g OGTTs in the morning and evening; the results of these tests indicated that glucose metabolism was significantly higher in the morning than in the evening (Fig. 2). This finding supports previous reports showing higher glucose tolerance in the morning than in the evening in healthy participants (Jarrett and Keen 1969; Jarrett et al. 1972; Carroll and Nestel 1973; Aparicio et al. 1974; Zimmet et al. 1974). The mechanism underlying diurnal variation in insulin sensitivity has been suggested to involve an increase in the levels of circulating free fatty acids in the evening (Yoshino et al. 2014).

Regarding glucose tolerance, previous studies of nocturnal rodents have shown that the central circadian clock in the suprachiasmatic nucleus ( $\mathrm{SCN}$ ) of the anterior hypothalamus is responsible for the 24-h rhythm in plasma glucose concentration (La Fleur et al. 1999). Interestingly, postprandial glucose concentrations and insulin secretions in rats are higher during the dark period (active phase) than during the light period (rest phase), but this distinction is lost in rats with lesions of the SCN rats (la Fleur et al. 2001). As demonstrated in these rodent studies, both the SCN circadian clock and the pancreatic clock play important roles in generating and maintaining the daily variations in glucose metabolism. In humans, the autonomous circadian clock system and desynchronization rhythm between the SCN-driven circadian rhythm (e.g., circadian rhythms of melatonin) and behavioral cycles have been reported to affect glucose metabolism (Van Cauter et al. 1991; Morgan et al. 1998; Morris et al. 2015). Therefore, the diurnal variation of glucose metabolism (e.g., glucose tolerance in OGTTs and postprandial glucose metabolism) in humans seems to be regulated by the SCN circadian clock.

Interestingly, our results show that morning mastication decreases postprandial blood glucose concentrations (Fig. 2A, C) and increases insulin secretion at $30 \mathrm{~min}$ (Fig. 3B) and insulinogenic index as a marker of early-phase $\beta$-cell function (Fig. 3E) following consumption of a highcarbohydrate meal. To the best of our knowledge, this represents the first evidence that the effects of mastication on early-phase insulin secretion depends on the time of day. 
The results differed from our expectation that mastication could improve glucose metabolism with increasing insulin secretion both in morning and evening. It has been reported that mastication increased insulin secretion before and after food intake through an enhancement of the preabsorptive insulin response (Teff 2000) and an increase dietary induced thermogenesis (Marques-Lopes et al. 2003). Although previous studies, which examined the effect of mastication on metabolism (Zhu et al. 2014; Hamada et al. 2014, 2016) performed during in morning and afternoon not in the evening, could demonstrate that mastication increases postprandial glucose metabolism which is consistent with the present findings. However, further studies might be needed to clarify whether effects of mastication on the preabsorptive insulin response and dietary induced thermogenesis depend on time of day. In contrast to the present findings, Borvornparadorn et al. (2019) found that chewing 50 times in the morning did not affect postprandial glucose and insulin responses in healthy, lean, and overweight participants, relative to those chewing 15 times. The study cohort consisted of 21 men and 24 women, and the test meal comprised ad libitum ham and cheese sandwiches; these differences between that study and ours may have influenced the postprandial glucose and insulin responses (Frape et al. 1997; Robertson et al. 2002; Anderwald et al. 2011; Horie et al. 2018).

Mastication is the first step of mechanical digestion and promotes the mixing of food with salivary amylase. Previous studies showed that increased mastication raises the saliva flow rate, which positively correlates with salivary alpha-amylase activity (Mackie and Pangorn 1990). Mastication also increases the secretion of GLP1, a protein known to enhance glucose-stimulated insulin secretion (MacDonald et al. 2002). In the present study, mastication did not significantly affect either plasma amylase activity (Fig. 4A) or total GLP1 concentration (Fig. 4B); therefore, these factors are less likely to be the precise mechanism involved in morning mastication-enhanced early-phase insulin secretion.

As determined via the MEQ, all participants in our study had an intermediate chronotype. Further studies are needed to determine whether the effect of morning mastication on postprandial glucose metabolism is chronotypedependent.

The present study is limited by its small cohort of participants and its restriction to non-obese and non-diabetic men. To fully understand its clinical implications, further studies are needed to determine whether morning mastication improves the poor early-phase insulin secretion in obese and/or diabetic patients (Matsumoto et al. 1997; Gerich 2002; Mizuno et al. 2007). In addition, we did not measure the participants' energy expenditure or levels of appetite-related hormones such as ghrelin, leptin, cholecystokinin, or peptide YY, which may have influenced our results. Future studies should be conducted to clarify the association between mastication at different times of day and enhanced early-phase insulin secretion.

In conclusion, the present study demonstrated that morning mastication decreases postprandial glucose concentrations following a high-carbohydrate meal and is associated with enhanced early-phase insulin secretion (increase the insulinogenic index). The effect of mastication on postprandial glucose metabolism was dependent on the time of day. Clinically, impairment of early-phase insulin secretion (decrease the insulinogenic index) is considered an early marker of $\beta$-cell dysfunction and the development of type 2 diabetes (Matsumoto et al. 1997; Gerich 2002; Mizuno et al. 2007); the present findings suggest that interventions to increase morning mastication may improve insulin-mediated glucose metabolism, reducing the incidence of obesity and type 2 diabetes.

\section{Author Contributions}

Y.Y. designed research; A.S., Y.Y., and Y.O. conducted research; A.S. and Y.Y. analyzed data; Y.Y. wrote paper. A.S., Y.Y., Y.O. had primary responsibility for final content.

\section{Acknowledgments}

We thank Mr. Ifeanyichukwu Akuma, Ms. Chihiro Okuyama, and Dr. Noriko Matsuura for technical assistance.

This work was partly supported by Grants in Aid for Scientific Research (C) from the Japan Society for the Promotion of Science (16K01723 to Y.Y.) and scholarship donations from the Akiyama Life Science Foundation (to Y.Y. and Y.O.) and the Hitachi Hokkaido University Laboratory (to Y.Y.).

\section{Conflict and Interest}

The authors declare no conflict of interest.

\section{References}

Ahmed, M., Gannon, M.C. \& Nuttall, F.Q. (1976) Postprandial plasma glucose, insulin, glucagon and triglyceride responses to a standard diet in normal subjects. Diabetologia, 12, 61-67.

Anderwald, C., Gastaldelli, A., Tura, A., Krebs, M., PromintzerSchifferl, M., Kautzky-Willer, A., Stadler, M., DeFronzo, R.A., Pacini, G. \& Bischof, M.G. (2011) Mechanism and effects of glucose absorption during an oral glucose tolerance test among females and males. J. Clin. Endocrinol. Metab., 96, 515-524.

Aparicio, N.J., Puchulu, F.E., Gagliardino, J.J., Ruiz, M., Llorens, J.M., Ruiz, J., Lamas, A. \& De Miguel, R. (1974) Circadian variation of the blood glucose, plasma insulin and human growth hormone levels in response to an oral glucose load in normal subjects. Diabetes, 23, 132-137.

Borvornparadorn, M., Sapampai, V., Champakerdsap, C., Kurupakorn, W. \& Sapwarobol, S. (2019) Increased chewing reduces energy intake, but not postprandial glucose and insulin, in healthy weight and overweight young adults. Nutr. Diet., 76, 89-94.

Carroll, K.F. \& Nestel, P.J. (1973) Diurnal variation in glucose tolerance and in insulin secretion in man. Diabetes, 22, 333-348.

Cassady, B.A., Hollis, J.H., Fulford, A.D., Considine, R.V. \& Mattes, R.D. (2009) Mastication of almonds: effects of lipid bioaccessibility, appetite, and hormone response. Am. J. Clin. Nutr., 89, 794-800.

Doi, Y., Minowa, M., Uchiyama, M., Okawa, M., Kim, K., Shibui, K. \& Kamei, Y. (2000) Psychometric assessment of subjective sleep quality using the Japanese version of the Pittsburgh 
Sleep Quality Index (PSQI-J) in psychiatric disordered and control subjects. Psychiatry Res., 97, 165-172.

Frape, D.L., Williams, N.R., Scriven, A.J., Palmer, C.R., O'Sullivan, K. \& Fletcher, R.J. (1997) Effects of high- and low-fat meals on the diurnal response of plasma lipid metabolite concentrations in healthy middle-aged volunteers. $\mathrm{Br} . \mathrm{J}$. Nutr., 77, 375-390.

Gerich, J.E. (2002) Is reduced first-phase insulin release the earliest detectable abnormality in individuals destined to develop type 2 diabetes? Diabetes, 51, S117-S121.

Hamada, Y., Kashima, H. \& Hayashi, N. (2014) The number of chews and meal duration affect diet-induced thermogenesis and splanchnic circulation. Obesity (Silver Spring), 22, E62-69.

Hamada, Y., Miyaji, A. \& Hayashi, N. (2016) Effect of postprandial gum chewing on diet-induced thermogenesis. Obesity (Silver Spring), 24, 878-885.

Hetherington, M.M. \& Boyland, E. (2007) Short-term effects of chewing gum on snack intake and appetite. Appetite, 48, 397-401.

Hetherington, M.M. \& Regan, M.F. (2011) Effects of chewing gum on short-term appetite regulation in moderately restrained eaters. Appetite, 57, 475-482.

Horie, I., Abiru, N., Eto, M., Sako, A., Akeshima, J., Nakao, T., Nakashima, Y., Niri, T., Ito, A., Nozaki, A., Haraguchi, A., Akazawa, S., Mori, Y., Ando, T. \& Kawakami, A. (2018) Sex differences in insulin and glucagon responses for glucose homeostasis in young healthy Japanese adults. J. Diabetes Investig., 9, 1283-1287.

Horne, J.A. \& Ostberg, O. (1976) A self-assessment questionnaire to determine morningness-eveningness in human circadian rhythms. Int. J. Chronobiol., 4, 97-110.

Jarrett, R.J., Baker, I.A., Keen, H. \& Oakley, N.W. (1972) Diurnal variation in oral glucose tolerance: blood sugar and plasma insulin levels morning, afternoon, and evening. Br. Med. J., 1, 199-201.

Jarrett, R.J. \& Keen, H. (1969) Diurnal variation of oral glucose tolerance: a possible pointer to the evolution of diabetes mellitus. Br. Med. J., 2, 341-344.

Komai, N., Motokubota, N., Suzuki, M., Hayashi, I., Moritani, T. \& Nagai, N. (2016) Thorough mastication prior to swallowing increases postprandial satiety and the thermic effect of a meal in young women. J. Nutr. Sci. Vitaminol. (Tokyo), 62, 288-294.

La Fleur, S.E., Kalsbeek, A., Wortel, J. \& Buijs, R.M. (1999) A suprachiasmatic nucleus generated rhythm in basal glucose concentrations. J. Neuroendocrinol., 11, 643-652.

la Fleur, S.E., Kalsbeek, A., Wortel, J., Fekkes, M.L. \& Buijs, R.M. (2001) A daily rhythm in glucose tolerance: a role for the suprachiasmatic nucleus. Diabetes, 50, 1237-1243.

Li, J., Zhang, N., Hu, L., Li, Z., Li, R., Li, C. \& Wang, S. (2011) Improvement in chewing activity reduces energy intake in one meal and modulates plasma gut hormone concentrations in obese and lean young Chinese men. Am. J. Clin. Nutr., 94, 709-716.

MacDonald, P.E., El-Kholy, W., Riedel, M.J., Salapatek, A.M., Light, P.E. \& Wheeler, M.B. (2002) The multiple actions of GLP-1 on the process of glucose-stimulated insulin secretion. Diabetes, 51 Suppl 3, S434-442.

Mackie, D.A. \& Pangborn, R.M. (1990) Mastication and its influence on human salivary flow and alpha-amylase secretion. Physiol. Behav., 47, 593-595.

Marques-Lopes, I., Forga, L. \& Martinez, J.A. (2003) Thermogenesis induced by a high-carbohydrate meal in fasted lean and overweight young men: insulin, body fat, and sympathetic nervous system involvement. Nutrition, 19, 25-29.

Maruyama, K., Sato, S., Ohira, T., Maeda, K., Noda, H., Kubota, Y., Nishimura, S., Kitamura, A., Kiyama, M., Okada, T., Imano, H., Nakamura, M., Ishikawa, Y., Kurokawa, M.,
Sasaki, S., et al. (2008) The joint impact on being overweight of self reported behaviours of eating quickly and eating until full: cross sectional survey. $B M J, 337$, a 2002 .

Matsumoto, K., Miyake, S., Yano, M., Ueki, Y., Yamaguchi, Y., Akazawa, S. \& Tominaga, Y. (1997) Glucose tolerance, insulin secretion, and insulin sensitivity in nonobese and obese Japanese subjects. Diabetes Care, 20, 1562-1568.

Mattes, R.D. \& Considine, R.V. (2013) Oral processing effort, appetite and acute energy intake in lean and obese adults. Physiol. Behav., 120, 173-181.

Mizuno, A., Arai, H., Fukaya, M., Sato, M., Hisami, Y.O., Takeda, E. \& Doi, T. (2007) Early-phase insulin secretion is disturbed in obese subjects with glucose intolerance. Metabolism, 56, 856-862.

Morgan, L., Arendt, J., Owens, D., Folkard, S., Hampton, S., Deacon, S., English, J., Ribeiro, D. \& Taylor, K. (1998) Effects of the endogenous clock and sleep time on melatonin, insulin, glucose and lipid metabolism. J. Endocrinol., 157, 443-451.

Morris, C.J., Yang, J.N., Garcia, J.I., Myers, S., Bozzi, I., Wang, W., Buxton, O.M., Shea, S.A. \& Scheer, F.A. (2015) Endogenous circadian system and circadian misalignment impact glucose tolerance via separate mechanisms in humans. Proc. Natl. Acad. Sci. USA, 112, E2225-2234.

Nuttall, F.Q., Gannon, M.C., Wald, J.L. \& Ahmed, M. (1985) Plasma glucose and insulin profiles in normal subjects ingesting diets of varying carbohydrate, fat, and protein content. J. Am. Coll. Nutr., 4, 437-450.

Ohkuma, T., Fujii, H., Iwase, M., Kikuchi, Y., Ogata, S., Idewaki, Y., Ide, H., Doi, Y., Hirakawa, Y., Mukai, N., Ninomiya, T., Uchida, K., Nakamura, U., Sasaki, S., Kiyohara, Y., et al. (2013) Impact of eating rate on obesity and cardiovascular risk factors according to glucose tolerance status: the Fukuoka Diabetes Registry and the Hisayama Study. Diabetologia, 56, 70-77.

Robertson, M.D., Jackson, K.G., Fielding, B.A., Williams, C.M. \& Frayn, K.N. (2002) Acute effects of meal fatty acid composition on insulin sensitivity in healthy post-menopausal women. Br. J. Nutr., 88, 635-640.

Rothwell, N.J. \& Stock, M.J. (1981) A role for insulin in the dietinduced thermogenesis of cafeteria-fed rats. Metabolism, 30, 673-678.

Seltzer, H.S., Allen, E.W., Herron, A.L. Jr. \& Brennan, M.T. (1967) Insulin secretion in response to glycemic stimulus: relation of delayed initial release to carbohydrate intolerance in mild diabetes mellitus. J. Clin. Invest., 46, 323-335.

Sonnier, T., Rood, J., Gimble, J.M. \& Peterson, C.M. (2014) Glycemic control is impaired in the evening in prediabetes through multiple diurnal rhythms. J. Diabetes Complications, 28, 836-843.

Suzuki, H., Fukushima, M., Okamoto, S., Takahashi, O., Shimbo, T., Kurose, T., Yamada, Y., Inagaki, N., Seino, Y. \& Fukui, T. (2005) Effects of thorough mastication on postprandial plasma glucose concentrations in nonobese Japanese subjects. Metabolism, 54, 1593-1599.

Takayama, S., Akamine, Y., Okabe, T., Koya, Y., Haraguchi, M., Miyata, Y., Sakai, T., Sakura, H. \& Sasaki, T. (2002) Rate of eating and body weight in patients with type 2 diabetes or hyperlipidaemia. J. Int. Med. Res., 30, 442-444.

Teff, K. (2000) Nutritional implications of the cephalic-phase reflexes: endocrine responses. Appetite, 34, 206-213.

Van Cauter, E., Blackman, J.D., Roland, D., Spire, J.P., Refetoff, S. \& Polonsky, K.S. (1991) Modulation of glucose regulation and insulin secretion by circadian rhythmicity and sleep. $J$. Clin. Invest., 88, 934-942.

Yamane, M., Ekuni, D., Mizutani, S., Kataoka, K., SakumotoKataoka, M., Kawabata, Y., Omori, C., Azuma, T., Tomofuji, T., Iwasaki, Y. \& Morita, M. (2014) Relationships between eating quickly and weight gain in Japanese university students: 
a longitudinal study. Obesity (Silver Spring), 22, 2262-2266.

Yoshino, J., Almeda-Valdes, P., Patterson, B.W., Okunade, A.L., Imai, S., Mittendorfer, B. \& Klein, S. (2014) Diurnal variation in insulin sensitivity of glucose metabolism is associated with diurnal variations in whole-body and cellular fatty acid metabolism in metabolically normal women. J. Clin. Endocrinol. Metab., 99, E1666-1670.

Zhu, Y. \& Hollis, J.H. (2014) Increasing the number of chews before swallowing reduces meal size in normal-weight, over- weight, and obese adults. J. Acad. Nutr. Diet., 114, 926-931.

Zhu, Y., Hsu, W.H. \& Hollis, J.H. (2014) Increased number of chews during a fixed-amount meal suppresses postprandial appetite and modulates glycemic response in older males. Physiol. Behav., 133, 136-140.

Zimmet, P.Z., Wall, J.R., Rome, R., Stimmler, L. \& Jarrett, R.J. (1974) Diurnal variation in glucose tolerance: associated changes in plasma insulin, growth hormone, and non-esterified fatty acids. Br. Med. J., 1, 485-488. 